\title{
Rapid improvement in vitamin D status with dietary 25-hydroxycholecalciferol in vitamin D insufficient dogs
}

\author{
Rachel A. Kurzbard ${ }^{1}$, Robert C. Backus ${ }^{1}$ and Shiguang $\mathrm{Yu}^{2}$ \\ ${ }^{1}$ Department of Veterinary Medicine and Surgery, College of Veterinary Medicine, University of Missouri, Columbia, MO 65211, USA \\ ${ }^{2}$ DSM Nutritional Products, LLC, Parsippany, NJ 07054, USA
}

(Received 12 October 2020 - Final revision received 16 January 2021 - Accepted 25 January 2021)

Journal of Nutritional Science (2021), vol. 10, e12, page 1 of 10

doi: $10.1017 /$ jns. 2021.4

Abstract

Vitamin D insufficiency is associated with various disease processes. We determined whether consumption of a diet supplemented with $\mathrm{HyD}^{\circledR}$, a 25 -hydroxycholecalciferol $\left(25(\mathrm{OH}) \mathrm{D}_{3}\right)$ source, would safely increase plasma $25(\mathrm{OH}) \mathrm{D}_{3}$ concentrations in Golden Retrievers with low vitamin D status. We hypothesised that dietary supplementation with $\mathrm{HyD}^{\circledR}$ would rapidly increase and sustain plasma $25(\mathrm{OH}) \mathrm{D}_{3}$ levels in healthy Golden Retrievers with low vitamin $\mathrm{D}$ status compared with supplementation with vitamin $\mathrm{D}_{3}$. Of fifty-seven privately owned dogs recruited with written owner consent, eighteen dogs with low vitamin D status were identified and sorted between two groups to have similar initial plasma $25(\mathrm{OH}) \mathrm{D}_{3}$ concentrations, sex distributions, ages and body weights. Dogs of each group were fed a dry dog food supplemented with either $16 \mu \mathrm{g} / \mathrm{kg}$ of $25(\mathrm{OH}) \mathrm{D}_{3}$ as $\mathrm{HyD}^{\circledR}(n 10)$ or $81 \mu \mathrm{g} / \mathrm{kg}$ of cholecalciferol $\left(\mathrm{D}_{3}\right)$ $\left(n\right.$ 8) for 4 months. Plasma $25(\mathrm{OH}) \mathrm{D}_{3}$ concentrations were determined monthly. A significant time effect $(P<0 \cdot 001)$ and time by group interaction $(P=0.0045)$ were found for monthly determined plasma $25(\mathrm{OH}) \mathrm{D}_{3}$ concentrations. Dogs fed the $\mathrm{HyD}^{\circledR}$-supplemented diet experienced a $40 \cdot 5 \%$ rise in plasma $25(\mathrm{OH}) \mathrm{D}_{3}$ values after 1 month $(P<0 \cdot 001)$ and no change thereafter. Plasma $25(\mathrm{OH}) \mathrm{D}_{3}$ values of dogs supplemented with vitamin $\mathrm{D}_{3}$ did not increase $(P>0 \cdot 05)$ and were less than values of dogs supplemented with $\operatorname{HyD}^{\circledR}(P=0 \cdot 044)$. With few exceptions, average haematologic, biochemical and urinalyses results remained within the reference range for both groups. Dietary supplementation with $\mathrm{HyD}^{\circledR}$ is sufficient to safely increase and sustain plasma $25(\mathrm{OH}) \mathrm{D}_{3}$ levels in healthy dogs.

Key words: Canine: Calcifediol: Cholecalciferol: 25-hydroxycholecalciferol: $\mathbf{H y D}^{\circledR}$

\section{Introduction}

Vitamin D is essential for numerous metabolic functions in dogs and other species. Unlike man, dogs are unable to use ultraviolet rays to synthesise sufficient vitamin D in the skin to meet their requirement ${ }^{(1)}$. Dogs rely on their diet to fulfil the vast majority of their vitamin $\mathrm{D}$ requirement. Vitamin $\mathrm{D}$ is most commonly known for its role in maintaining calcium and phosphorus homeostasis. Vitamin D also plays numerous key roles via receptors on target cells to modulate immune and cardiovascular function ${ }^{(2-4)}$.

The best indicator of vitamin $\mathrm{D}$ status is plasma 25-hydroxyvitamin D $(25(\mathrm{OH}) \mathrm{D})$, which is the most abundant circulating metabolite of vitamin $\mathrm{D}^{(4)}$. Adequate intake levels of vitamin $\mathrm{D}$ in dogs were recommended by the National Research Council (NRC), based on the amount required to prevent bone pathologies in growing puppies ${ }^{(5)}$. These values have been extended to adult dogs. However, the vitamin D requirements for normal bone growth in puppies may be different from the concentrations required for optimal health and prevention of disease related to insufficiency in adult dogs.

The definition of vitamin D sufficiency in dogs has remained controversial. In man, levels of $25(\mathrm{OH}) \mathrm{D}$ from $20-50 \mathrm{ng} / \mathrm{ml}$ are required to prevent skeletal abnormalities ${ }^{(6)}$. These levels are controversial due to inconsistent protocols for plasma $25(\mathrm{OH}) \mathrm{D}$ determination. Recently, a plasma 25-hydroxyvitamin D3 (25(OH) $\left.\mathrm{D}_{3}\right)$ level of $100-120 \mathrm{ng} / \mathrm{ml}$

* Corresponding author: Rachel A. Kurzbard, fax (949) 833-7530, email rakurzbard@gmail.com

(C) The Author(s), 2021. Published by Cambridge University Press on behalf of The Nutrition Society. This is an Open Access article, distributed under the terms of the Creative Commons Attribution licence (http://creativecommons.org/licenses/by/4.0/), which permits unrestricted re-use, distribution, and reproduction in any medium, provided the original work is properly cited. 
was considered sufficient for dogs, based on the minimisation of variance of parathyroid hormone and mean C-reactive protein levels at this range ${ }^{(7)}$. According to this data, many apparently healthy dogs may be vitamin $\mathrm{D}$ insufficient despite most commercial pet foods having adequate vitamin $\mathrm{D}$ levels ${ }^{(8)}$. Vitamin D insufficiency in dogs has been associated with a host of various disease processes ${ }^{(3,7,9-13)}$. Whether insufficient vitamin D status is a consequence of disease, or risk factor for disease is presently under investigation. Several studies have indicated a correlation between low vitamin D status and disease, with insufficient vitamin $\mathrm{D}$ status corresponding to a poorer prognosis ${ }^{(7,14,15)}$. To combat the potential for deficiency contributing to disease, appropriate supplementation is warranted.

Supplementation with excess vitamin D in the form of cholecalciferol (D3) did not improve plasma D status in privately owned dogs that were considered vitamin $\mathrm{D}$ insufficient ${ }^{(16)}$. However, supplementing $25(\mathrm{OH}) \mathrm{D}_{3}$ rapidly improved plasma D status in research $\operatorname{dogs}{ }^{(17)}$. A similar improvement was found in healthy people, and in people with renal disease $\mathrm{e}^{(18-20)}$.

The objective of the present study was to determine whether consumption of a diet using vitamin $\mathrm{D}$ supplemented as $\mathrm{HyD}^{\circledR}$, a proprietary form of $25(\mathrm{OH}) \mathrm{D}_{3}$, would increase the plasma $25(\mathrm{OH}) \mathrm{D}_{3}$ of healthy dogs above that achievable with D3. Utilising one dog breed served to minimise observational variance from genetic heterogeneity. Golden Retrievers were chosen due to breed predisposition towards low vitamin D status ${ }^{(2)}$, as well as their predisposition to developing various neoplastic diseases and other diseases associated with vitamin $\mathrm{D}$ insufficiency ${ }^{(2,7,21)}$. Among dog breeds, Golden Retrievers have a high lifetime risk for the development of cancers $^{(22)}$. This makes them an ideal subject for a study evaluating the improvement of insufficient vitamin D. We hypothesised that supplementation with vitamin $\mathrm{D}$ in the form of 25 $(\mathrm{OH}) \mathrm{D}_{3}$ would rapidly and safely increase plasma $25(\mathrm{OH}) \mathrm{D}_{3}$ and thereby sustain an elevation in vitamin $\mathrm{D}$ status. We also hypothesised that the plasma $25(\mathrm{OH}) \mathrm{D}_{3}$ of dogs fed a diet supplemented with a high but not excessive amount of vitamin D3 would not significantly increase.

\section{Experimental methods}

Part I

Animals. All procedures were reviewed and approved by the institutional animal care and use committee (Protocol \#8908). Canine participants were recruited at a breed club show (Golden Retriever National Event, St. Louis, Missouri) and an announcement through an e-mail list-serve. A total of fifty-seven privately owned, adult dogs aged 2-10 years were recruited. Dogs were excluded if they were younger than 2 years or older than 10 years, if they had underlying disease processes, were pregnant, lactating, or had pregnancy planned during the trial duration. Dogs were also excluded if they were receiving vitamins or supplements that could impact vitamin D status. Signed informed consent forms were obtained from owners prior to $\operatorname{dog}$ evaluation and blood collection. This form relayed the purpose and expected outcomes of the study, and potential risks to the patient. Owners were informed of the potential for their dog to be enrolled in the second part of the study, pending vitamin $\mathrm{D}$ status.

Evaluation and sample collection. All dogs sampled were purebred Golden Retrievers (thirty-three males, twenty-four females). Body weights ranged from 23.5 to $40 \cdot 1 \mathrm{~kg}$. All weights were evaluated on a single scale. Approximately $6 \mathrm{ml}$ of venous blood was obtained from each dog via jugular or cephalic venipuncture. Blood was immediately transferred to a lithium heparinised tube and spun down in a centrifuge at $1200 \mathrm{~g}$ for $10 \mathrm{~min}$ within $30 \mathrm{~min}$ of collection. Plasma was harvested and stored in a separate plastic snap-cap tube in a $-20^{\circ} \mathrm{C}$ freezer until evaluation. Plasma $25(\mathrm{OH}) \mathrm{D}_{3}$ status of each animal was evaluated using a high-performance liquid chromatography (HPLC) method ${ }^{(23)}$ that was adapted for analysis of $\operatorname{dog}$ serum and plasma ${ }^{(16,17)}$.

\section{Part II}

Animals. Owners of dogs determined to have plasma 25 $(\mathrm{OH}) \mathrm{D}_{3}$ concentrations less than $50 \mathrm{ng} / \mathrm{ml}$ were notified by phone or e-mail and offered participation in the second part of the study: a diet supplementation trial to evaluate the efficacy of vitamin D supplementation using a custommanufactured, dry-expanded diet (Table 1). Owners of dogs with the lowest vitamin $\mathrm{D}$ status were contacted first, due to the association between vitamin $\mathrm{D}$ insufficiency and reduced odds of survival ${ }^{(14,24)}$.

Reviews of medical and dietary history of all participants were conducted to ensure that participating dogs were healthy. Complete diet histories were requested of all participants. Clinical laboratory variables (complete blood count and routine biochemical analyses of plasma and urine) were evaluated prior to participation. Physical examinations were conducted by primary veterinarians to ensure that canine participants were healthy prior to study commencement. Instructions were provided to owners of participating dogs to avoid alterations in the environment and exercise schedule during the supplementation trial.

Groups. Dogs were assigned to a control ( $n 9$ initial, $n 8$ completion) or treatment group ( $n$ 10), stratified based on initial plasma $25(\mathrm{OH}) \mathrm{D}_{3}$ status. Groups were balanced based on plasma $25(\mathrm{OH}) \mathrm{D}_{3}$ concentration, sex, body weight and group size as much as possible (Table 2). Dogs belonging to the same household were assigned to the same group. Owners were blinded as to which group their dogs were assigned.

Diet. Once groups were assigned, all dogs received one of two diets formulated in compliance with AAFCO dog food nutrient profiles for adult maintenance except for vitamin D3 (Table 1). The control diet provided vitamin D as D3 and the treatment diet provided vitamin $\mathrm{D}$ as $25(\mathrm{OH}) \mathrm{D}_{3}$ $\left(\mathrm{HyD}^{\circledR}{ }^{\circledR}\right.$, DSM Nutritional Products, LLC, New Jersey 
Table 1. Target and measured as-fed proximate nutrient contents and concentrations of calcium, phosphorus, D vitamers and metabolisable energy of trial diets $^{a}$

\begin{tabular}{|c|c|c|c|c|}
\hline \multirow[b]{2}{*}{ Nutrient } & \multicolumn{2}{|l|}{ D3 } & \multicolumn{2}{|l|}{$\mathrm{HyD}^{\circledR}$} \\
\hline & Target & Measured & Target & Measured \\
\hline Crude protein $\left(\%^{b}\right)$ & $39(\min )$ & $40 \cdot 3$ & $39(\min )$ & 39.5 \\
\hline Crude fat (\%) & 14 (min) & $11 \cdot 7$ & 14 (min) & $10 \cdot 7$ \\
\hline Crude fibre (\%) & $3(\max )$ & nd & $3(\max )$ & nd \\
\hline Moisture (\%) & $12(\max )$ & $6 \cdot 9$ & $12(\max )$ & $6 \cdot 6$ \\
\hline Calcium (\%) & - & 1.0 & - & 1.0 \\
\hline Phosphorus (\%) & - & 0.9 & - & 0.9 \\
\hline Vitamin D3 (IU/kg) & 3250 & 3630 & 0 & 70 \\
\hline 25-hydroxyvitamin D3 ( $\mu \mathrm{g} / \mathrm{kg})$ & - & $<1$ & 16 & 19 \\
\hline Metabolisable Energy (kcal/kg) & 3623 & - & 3623 & - \\
\hline
\end{tabular}

nd, Not determined.

${ }^{a}$ Diet ingredients listed by weight, as is, in decreasing order: Chicken by-product meal, maize gluten meal, dried egg product, brewers rice, maize, chicken fat, wheat, natural flavour, sorghum, dried plain beet pulp, potassium chloride, salt, dicalcium phosphate, pea fibre, calcium carbonate, vitamin $E$ supplement, niacin supplement, thiamine mononitrate, calcium pantothenate, vitamin A supplement, mineral oil, pyridoxine hydrochloride, riboflavin supplement, vitamin D3 supplement, vitamin B12 supplement, folic acid, bio-

tin, tocopherols, ferrous sulphate, zinc oxide, manganese oxide, copper sulphate, sodium selenite, cobalt carbonate, ethylenediamine dihydriodide, taurine, choline chloride.

${ }^{\mathrm{b}}$ Weight $(\mathrm{g}) / \mathrm{diet}$ weight $(100 \mathrm{~g})$.

07054). Proximate analysis of a random sample of treatment and control diets was performed at an external laboratory (University of Missouri Agricultural Experiment Station Chemical Laboratories). Owners of participating dogs were provided feeding instructions so that dogs consumed the test diet at the caloric equivalence of their historic diet to maintain their body weight. Dogs were transitioned to their new diet over a 7 -d period.

Plasma collection. Canine participant owners and veterinary clinics listed by the owners were e-mailed instructions on monthly appointments for blood collection. Blood samples were collected by the author (R. K.) or by the participant's primary veterinary clinic, depending on dog location. Samples were centrifuged and plasma was harvested within $1 \mathrm{~h}$ of sample collection. Plasma was shipped overnight on ice packs to the university. Upon arrival, plasma was placed in an airtight, freezer-resistant plastic tube and stored for a maximum of 4 months at $-20^{\circ} \mathrm{C}$ for batch analysis of plasma $25(\mathrm{OH}) \mathrm{D}_{3}$ concentrations. Tubes were labelled so that the identity of the sample was not revealed to the personnel measuring plasma $25(\mathrm{OH}) \mathrm{D}_{3}$. Blood collections were repeated at weeks 4,8 and 16 for assessment vitamin $\mathrm{D}$ status from plasma $25(\mathrm{OH}) \mathrm{D}_{3}$ concentrations. Plasma $25(\mathrm{OH}) \mathrm{D}_{3}$ analysis was repeated on all plasma samples at completion of the study to reduce inter-assay variance. Upon completion of the trial, at week 16, all $\operatorname{dogs}$ had repeated clinical laboratory analysis of blood, plasma and urine.

Laboratory analyses. Clinical haematology (Sysmex XT-2000i; Sysmex America, Inc., Lincolnshire, IL), plasma biochemistry (Beckman AU480, Beckman Coulter, Inc., Brea, CA) and urinalyses (Clinitek Status, Siemens Medical Solutions USA, Inc., Malvern, PA) were performed at the University of Missouri Veterinary Medical Diagnostic Laboratory, Columbia, MO. The same analysers were used for all samples. Plasma $25(\mathrm{OH}) \mathrm{D}_{3}$ of each animal was evaluated monthly and batch-evaluated at the end of the experiment to minimise variability using the chromatographic method as described earlier.

Statistical analysis. Sample size power analyses were performed to determine the number of dogs required in a parallel study to demonstrate the treatment effect. The power analysis assumed a standard deviation of plasma $25(\mathrm{OH}) \mathrm{D}_{3}$ between dogs to be $14 \mathrm{ng} / \mathrm{ml}$. The analysis indicated that a minimum of $46 \%$ difference in mean plasma $25(\mathrm{OH}) \mathrm{D}_{3}$ concentration between groups, seven dogs would be required to find significance at $\alpha=0.05$ with the power of $\beta=0 \cdot 8$.

Statistical analyses were performed using statistical software $\left(\mathrm{SAS}^{\circledR}\right.$ Version 9.4; SAS Institute, Cary, NC). Data were tested

Table 2. Characteristics and vitamin $\mathrm{D}$ status of dogs enrolled in the control (D3) and treatment $\left(\mathrm{HyD}^{\circledR}\right)$ groups at the beginning of the diet supplementation trial

\begin{tabular}{|c|c|c|c|c|c|}
\hline & \multicolumn{2}{|l|}{ D3 } & \multicolumn{2}{|l|}{$\mathrm{HyD}^{\circledR}$} & \\
\hline & Median & Range & Median & Range & \\
\hline Number & 8 & - & 10 & - & \\
\hline Sex (female \%) & 63 & - & 60 & - & $\mathrm{NS}^{*}$ \\
\hline Age (years) & $7 \cdot 5$ & $2-10$ & $4 \cdot 0$ & $2-10$ & $\mathrm{NS}^{\dagger}$ \\
\hline Body weight $(\mathrm{kg})$ & $28 \cdot 2$ & $23 \cdot 5-31 \cdot 2$ & $26 \cdot 9$ & $24 \cdot 5-36 \cdot 6$ & $\mathrm{NS}^{\dagger}$ \\
\hline Plasma 25-hydroxyvitamin D (ng/ml) & $25 \cdot 3$ & $13 \cdot 9-36 \cdot 6$ & $25 \cdot 6$ & $17 \cdot 7-34 \cdot 7$ & $\mathrm{NS}^{\dagger}$ \\
\hline
\end{tabular}


for normality using skew and kurtosis measures, and visual inspection of plots. Non-normally distributed data was log-transformed. Normally distributed biochemical and haematologic observations were analysed with paired $t$ tests. Non-normally distributed data and categorical results were evaluated using Wilcoxon rank-sign testing. A repeatedmeasures, mixed models ANOVA that employed TukeyKramer adjusted post hoc multiple comparisons were used to determine the significance of differences between treatment group outcomes at each blood sampling time, i.e. initial and at 1, 2, 3 and 4 months after the D-vitamer supplementations. Urine calcium/creatinine and urine phosphorus/creatinine ratios were evaluated using paired $t$ tests. The significance of D-vitamer supplementation type on frequencies of categorical urinalysis findings was determined with Fisher's exact tests. $P$-values $\leq 0.05$ were considered significant.

\section{Results}

\section{Initial survey}

In the initial cohort of fifty-seven dogs, plasma $25(\mathrm{OH}) \mathrm{D}_{3}$ concentrations ranged from 14 to $59 \mathrm{ng} / \mathrm{ml}$. Fifty-three dogs $(91.4 \%)$ had $25(\mathrm{OH}) \mathrm{D}_{3}$ values below $50 \mathrm{ng} / \mathrm{ml}$. Concentrations were normally distributed. Mean and median concentrations were $34 \mathrm{ng} / \mathrm{ml}$. Twenty-six dogs (45\%) had $25(\mathrm{OH}) \mathrm{D}_{3}$ concentrations below $33 \mathrm{ng} / \mathrm{ml}$.

\section{Group parameters}

Initial veterinary examinations indicated that all participants were healthy. Initial laboratory parameters evaluated on participants of part II were within the laboratory reference ranges, with few exceptions (Tables 3-5). Sex distribution, body weight, ages and plasma $25(\mathrm{OH}) \mathrm{D}_{3}$ concentrations of both groups were similar (Table 2 ).

\section{Laboratory values}

Clinical haematology and biochemistry values and urinalyses parameters at the trial end were not significantly different between groups. There were a few significant differences between pre- and post-trial values within groups (Tables 35). At the trial end, the means of erythrocyte counts, haemoglobin concentrations and haematocrits of dogs of the $\mathrm{HyD}^{\circledR}$

Table 3. Plasma clinical haematology variable medians and ranges of dogs before and 4 months after diet supplementation with vitamin D3 $(n 8)$ or HyD ${ }^{\circledR}(n$ 10)

\begin{tabular}{|c|c|c|c|c|c|c|c|c|c|}
\hline & \multicolumn{6}{|c|}{ Supplementation } & & & \multirow{3}{*}{$\begin{array}{l}\text { Reference } \\
\text { interval }\end{array}$} \\
\hline & \multicolumn{2}{|l|}{ Vitamin D3 } & \multirow[b]{2}{*}{$P^{\star}$} & \multicolumn{2}{|l|}{$\mathrm{HyD}^{\circledR}$} & \multirow[b]{2}{*}{$P^{*}$} & \multicolumn{2}{|c|}{ Vitamin $\mathrm{D} 3$ v. HyD ${ }^{\circledR}$} & \\
\hline & Pre-trial & 4 months & & Pre-trial & 4 months & & Pre-trialP & 4 months $P$ & \\
\hline 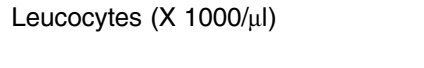 & $\begin{array}{r}9.1 \\
{[5.9-11.0]}\end{array}$ & $\begin{array}{r}9.2 \\
{[6 \cdot 7-11 \cdot 7]}\end{array}$ & 0.92 & $\begin{array}{r}8 \cdot 2 \\
{[5 \cdot 2-11 \cdot 7]}\end{array}$ & $\begin{array}{r}8 \cdot 1 \\
{[5 \cdot 2-12 \cdot 2]}\end{array}$ & 0.83 & 0.29 & 0.38 & $4 \cdot 1-14 \cdot 6$ \\
\hline 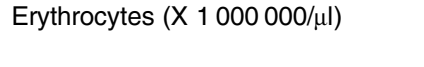 & $\begin{array}{r}7 \cdot 1 \\
{[6 \cdot 2-8 \cdot 2]}\end{array}$ & $\begin{array}{r}6 \cdot 8 \\
{[6 \cdot 3-7 \cdot 1]}\end{array}$ & 0.14 & $\begin{array}{r}7 \cdot 1 \\
{[5 \cdot 5-8]}\end{array}$ & $\begin{array}{r}6 \cdot 6 \\
{[5 \cdot 8-7 \cdot 7]}\end{array}$ & 0.01 & 0.69 & 0.94 & $5 \cdot 3-8 \cdot 3$ \\
\hline Haemoglobin (g/dl) & $\begin{array}{r}16 \cdot 6 \\
{[15-20 \cdot 6]}\end{array}$ & $\begin{array}{r}16 \cdot 3 \\
{[15-17 \cdot 8]}\end{array}$ & 0.24 & $\begin{array}{r}17 \\
{[13 \cdot 7-19 \cdot 7]}\end{array}$ & $\begin{array}{r}16 \cdot 4 \\
{[14 \cdot 4-19 \cdot 2]}\end{array}$ & 0.01 & 0.93 & 0.99 & $13 \cdot 3-20 \cdot 8$ \\
\hline Haematocrit (\%) & $\begin{array}{r}48 \cdot 2 \\
{[42 \cdot 9-61 \cdot 1]}\end{array}$ & $\begin{array}{r}48 \cdot 2 \\
{[44 \cdot 2-52 \cdot 2]}\end{array}$ & 0.38 & $\begin{array}{r}49.7 \\
{[42-57.3]}\end{array}$ & $\begin{array}{r}48.5 \\
{[43.5-53.3]}\end{array}$ & 0.01 & 0.91 & 0.96 & $37 \cdot 2-56 \cdot 4$ \\
\hline $\mathrm{MCV}^{\dagger}(\mathrm{fl})$ & $\begin{array}{r}69 \\
{[66 \cdot 8-74.8]}\end{array}$ & $\begin{array}{r}73 \cdot 1 \\
{[66 \cdot 6-77.5]}\end{array}$ & 0.04 & $\begin{array}{r}70 \cdot 6 \\
{[67.3-76.9]}\end{array}$ & $\begin{array}{r}71 \cdot 1 \\
{[67.9-81.4]}\end{array}$ & 0.33 & 0.57 & 0.69 & $62 \cdot 5-72 \cdot 9$ \\
\hline $\mathrm{MCHgb}^{\ddagger}(\mathrm{pg})$ & $\begin{array}{r}24.2 \\
{[22 \cdot 9-25 \cdot 8]}\end{array}$ & $\begin{array}{r}25.0 \\
{[22.9-26]}\end{array}$ & 0.08 & $\begin{array}{r}24 \cdot 6 \\
{[23 \cdot 3-26 \cdot 4]}\end{array}$ & $\begin{array}{r}24.7 \\
{[23.5-26.4]}\end{array}$ & 0.73 & 0.45 & 0.96 & $22 \cdot 4-26 \cdot 2$ \\
\hline $\mathrm{MCHC}^{\S}(\mathrm{g} / \mathrm{dl})$ & $\begin{array}{r}34 \cdot 3 \\
{[33.7-35 \cdot 5]}\end{array}$ & $\begin{array}{r}34 \cdot 2 \\
{[33 \cdot 3-34 \cdot 6]}\end{array}$ & 0.21 & $\begin{array}{r}34 \cdot 5 \\
{[32 \cdot 6-36 \cdot 1]}\end{array}$ & $\begin{array}{r}34 \cdot 1 \\
{[32 \cdot 5-36 \cdot 6]}\end{array}$ & 0.35 & 0.76 & 0.51 & $34 \cdot 2-37 \cdot 9$ \\
\hline Platelets $(X 1000 / \mu l)$ & $\begin{array}{r}283 \\
{[220-399]}\end{array}$ & $\begin{array}{r}289 \\
{[247-465]}\end{array}$ & $0 \cdot 15$ & $\begin{array}{r}256 \\
{[174-450]}\end{array}$ & $\begin{array}{r}278 \\
{[150-477]}\end{array}$ & 0.23 & 0.39 & 0.47 & $140-350$ \\
\hline Segmented neutrophils (X 1000/ul) & $\begin{array}{r}5 \cdot 21 \\
{[3 \cdot 11-7 \cdot 72]}\end{array}$ & $\begin{array}{r}5.45 \\
{[3.21-7.63]}\end{array}$ & 0.96 & $\begin{array}{r}4.65 \\
{[2.79-8.05]}\end{array}$ & $\begin{array}{r}4.30 \\
{[2.14-8.19]}\end{array}$ & 0.65 & 0.32 & 0.24 & $2 \cdot 27-10 \cdot 60$ \\
\hline 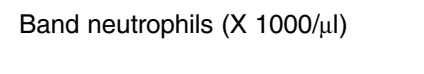 & $\begin{array}{r}0.06 \\
{[0.00-0.28]}\end{array}$ & $\begin{array}{r}0.12 \\
{[0.00-0.35]}\end{array}$ & 0.63 & $\begin{array}{r}0.03 \\
{[0.00-0.09]}\end{array}$ & $\begin{array}{r}0.04 \\
{[0.00-0.17]}\end{array}$ & 0.49 & 0.36 & 0.24 & $0.00-0.18$ \\
\hline Lymphocytes (X 1000/ul) & $\begin{array}{r}2.70 \\
{[1.88-3 \cdot 14]}\end{array}$ & $\begin{array}{r}2.58 \\
{[1.4-3.62]}\end{array}$ & 0.94 & $\begin{array}{r}2.34 \\
{[1.3-3.72]}\end{array}$ & $\begin{array}{r}2.67 \\
{[2.06-4.97]}\end{array}$ & 0.24 & 0.40 & 0.67 & $0 \cdot 83-4 \cdot 80$ \\
\hline Monocytes (X 1000/ $\mu \mathrm{l})$ & $\begin{array}{r}0.30 \\
{[0.18-1 \cdot 10]}\end{array}$ & $\begin{array}{r}0.31 \\
{[0.20-0.58]}\end{array}$ & 0.59 & $\begin{array}{r}0.20 \\
{[0.10-0.60]}\end{array}$ & $\begin{array}{r}0.21 \\
{[0.10-0.64]}\end{array}$ & 0.56 & $0 \cdot 12$ & 0.35 & $0.05-1 \cdot 24$ \\
\hline 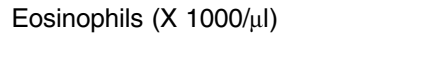 & $\begin{array}{r}0.31 \\
{[0.11-0.98]}\end{array}$ & $\begin{array}{r}0.39 \\
{[0-1.17]}\end{array}$ & 0.28 & $\begin{array}{r}0.45 \\
{[0.08-1.33]}\end{array}$ & $\begin{array}{r}0.45 \\
{[0.21-1.06]}\end{array}$ & 0.54 & 0.46 & 0.63 & $0.07-1.40$ \\
\hline 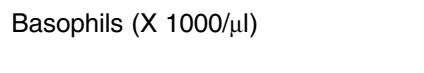 & $\begin{array}{r}0 \\
{[0-0]}\end{array}$ & $\begin{array}{r}0 \\
{[0-0]}\end{array}$ & & $\begin{array}{r}0 \\
{[0-0]}\end{array}$ & $\begin{array}{r}0 \\
{[0-0]}\end{array}$ & & & & $\mathrm{nr}^{\dagger}$ \\
\hline Nucleated erythrocytes & $\begin{array}{r}0 \\
{[0-0]}\end{array}$ & $\begin{array}{r}0 \\
{[0-0]}\end{array}$ & & $\begin{array}{r}0 \\
{[0-1]}\end{array}$ & $\begin{array}{r}0 \\
{[0-1]}\end{array}$ & & & & $0-1$ \\
\hline
\end{tabular}

${ }^{*} P$-values of comparisons between pre-trial and month 4 values.

${ }^{\dagger} \mathrm{MCV}$, Mean Corpuscular volume.

${ }^{\ddagger} \mathrm{MCHgb}$, mean corpuscular haemoglobin

$\S \mathrm{MCHC}$, Mean Corpuscular Haemoglobin Concentration. 
Table 4. Plasma clinical chemistry variable medians and ranges of dogs before and 4 months after diet supplementation with vitamin $\mathrm{D} 3(n 8)$ or HyD $\mathrm{D}^{\circledR}(n 10)$

\begin{tabular}{|c|c|c|c|c|c|c|c|c|c|}
\hline & \multicolumn{6}{|c|}{ Supplementation } & & & \multirow[b]{3}{*}{ Reference interval } \\
\hline & \multicolumn{2}{|l|}{ Vitamin D3 } & \multirow[b]{2}{*}{$P^{\star}$} & \multicolumn{2}{|l|}{$\mathrm{HyD}^{\circledR}$} & \multirow[b]{2}{*}{$P^{*}$} & \multicolumn{2}{|c|}{ Vitamin $\mathrm{D} 3$ v. HyD ${ }^{\circledR}$} & \\
\hline & Pre-trial & 4 months & & Pre-trial & 4 months & & Pre-trial $P$ & 4 months $P$ & \\
\hline Glucose (mol/l) & $\begin{array}{r}5.5 \\
{[5-6 \cdot 3]}\end{array}$ & $\begin{array}{r}5 \cdot 4 \\
{[4 \cdot 6-5 \cdot 7]}\end{array}$ & 0.95 & $\begin{array}{r}5 \cdot 4 \\
{[4 \cdot 6-5 \cdot 7]}\end{array}$ & $\begin{array}{r}5 \cdot 1 \\
{[4.9-5 \cdot 7]}\end{array}$ & 0.48 & 0.34 & $0 \cdot 17$ & $4 \cdot 5-6 \cdot 4$ \\
\hline Urea nitrogen (mg/dl) & $\begin{array}{r}16 \\
{[13-24]}\end{array}$ & $\begin{array}{r}21 \\
{[17-25]}\end{array}$ & 0.01 & $\begin{array}{r}17 \\
{[7-20]}\end{array}$ & $\begin{array}{r}20 \\
{[14-24]}\end{array}$ & 0.01 & 0.45 & 0.21 & $8-29$ \\
\hline Creatinine (mg/dl) & $\begin{array}{r}1.0 \\
{[0.7-1.2]}\end{array}$ & $\begin{array}{r}0.9 \\
{[0.7-0.9]}\end{array}$ & 0.03 & $\begin{array}{r}1.0 \\
{[0.8-1.3]}\end{array}$ & $\begin{array}{r}0.8 \\
{[0.7-0.9]}\end{array}$ & 0.01 & 0.40 & 0.39 & $0.7-1.4$ \\
\hline Sodium (meq/l) & $\begin{array}{r}147 \\
{[145-149]}\end{array}$ & $\begin{array}{r}147 \\
{[146-154]}\end{array}$ & 0.62 & $\begin{array}{r}147 \\
{[147-149]}\end{array}$ & $\begin{array}{r}147 \\
{[136-149]}\end{array}$ & 0.07 & 0.79 & 0.13 & $145-151$ \\
\hline Potassium (meq/l) & $\begin{array}{r}4.2 \\
{[3.9-4.4]}\end{array}$ & $\begin{array}{r}4.0 \\
{[3 \cdot 6-4.9]}\end{array}$ & 0.82 & $\begin{array}{r}4.1 \\
{[3.6-4 \cdot 3]}\end{array}$ & $\begin{array}{r}3.8 \\
{[3.4-4.3]}\end{array}$ & 0.34 & $0 \cdot 13$ & $0 \cdot 18$ & $3.5-4 \cdot 9$ \\
\hline Chloride (meq/l) & $\begin{array}{r}112 \\
{[110-116]}\end{array}$ & $\begin{array}{r}113 \\
{[111-116]}\end{array}$ & 0.56 & $\begin{array}{r}113.5 \\
{[112-116]}\end{array}$ & $\begin{array}{r}113 \\
{[106-117]}\end{array}$ & 0.57 & 0.22 & 0.87 & $110-117$ \\
\hline Bicarbonate (meq/l) & $\begin{array}{r}22 \\
{[17-26]}\end{array}$ & $\begin{array}{r}19.5 \\
{[14-21]}\end{array}$ & 0.01 & $\begin{array}{r}20 \\
{[18-24]}\end{array}$ & $\begin{array}{r}17.5 \\
{[15-20]}\end{array}$ & 0.01 & 0.25 & $0 \cdot 16$ & $17-26$ \\
\hline Anion Gap (meq/l) & $\begin{array}{r}17 \\
{[14-19]}\end{array}$ & $\begin{array}{r}19 \\
{[17-31]}\end{array}$ & 0.01 & $\begin{array}{r}18 \\
{[14-19]}\end{array}$ & $\begin{array}{r}20 \\
{[16-21]}\end{array}$ & 0.01 & 0.46 & 0.56 & $12-20$ \\
\hline Albumin (g/dl) & $\begin{array}{r}3 \cdot 0 \\
{[2 \cdot 8-3 \cdot 2]}\end{array}$ & $\begin{array}{r}3.0 \\
{[2 \cdot 7-3.4]}\end{array}$ & 1.00 & $\begin{array}{r}3 \cdot 1 \\
{[2 \cdot 8-3 \cdot 2]}\end{array}$ & $\begin{array}{r}3 \cdot 1 \\
{[2 \cdot 7-3 \cdot 2]}\end{array}$ & 0.34 & 0.37 & 0.74 & $2 \cdot 7-3 \cdot 7$ \\
\hline Total Protein (g/dl) & $\begin{array}{r}5 \cdot 7 \\
{[5 \cdot 5-6 \cdot 6]}\end{array}$ & $\begin{array}{r}6 \cdot 1 \\
{[5 \cdot 5-6 \cdot 8]}\end{array}$ & 0.51 & $\begin{array}{r}5.85 \\
{[5.5-6.4]}\end{array}$ & $\begin{array}{r}6.0 \\
{[5.4-9.6]}\end{array}$ & 0.49 & 0.62 & 0.39 & $5 \cdot 4-6 \cdot 9$ \\
\hline Globulin (g/dl) & $\begin{array}{r}2.8 \\
{[2.5-3.6]}\end{array}$ & $\begin{array}{r}3.3 \\
{[2.4-3.8]}\end{array}$ & 0.01 & $\begin{array}{r}2 \cdot 8 \\
{[2 \cdot 4-3 \cdot 2]}\end{array}$ & $\begin{array}{r}3.0 \\
{[2.5-3.4]}\end{array}$ & 0.12 & 0.74 & $0 \cdot 16$ & $2 \cdot 4-3 \cdot 7$ \\
\hline Calcium (mg/dl) & $\begin{array}{r}9.55 \\
{[9.3-9.8]}\end{array}$ & $\begin{array}{r}9.7 \\
{[9.5-10.8]}\end{array}$ & $0 \cdot 16$ & $\begin{array}{r}9.65 \\
{[9.2-10.2]}\end{array}$ & $\begin{array}{r}9.85 \\
{[9.3-10.5]}\end{array}$ & 0.47 & 0.34 & 0.56 & $9 \cdot 1-10 \cdot 8$ \\
\hline Phosphorus (mg/dl) & $\begin{array}{r}3.8 \\
{[3.4-4.4]}\end{array}$ & $\begin{array}{r}3.2 \\
{[2.7-3.5]}\end{array}$ & 0.02 & $\begin{array}{r}3 \cdot 8 \\
{[2 \cdot 1-4 \cdot 3]}\end{array}$ & $\begin{array}{r}3.5 \\
{[2.5-4.0]}\end{array}$ & 0.76 & 0.22 & 0.14 & $2 \cdot 3-5 \cdot 0$ \\
\hline Cholesterol (mg/dl) & $\begin{array}{r}217 \\
{[208-249]}\end{array}$ & $\begin{array}{r}252 \\
{[236-351]}\end{array}$ & 0.03 & $\begin{array}{r}245 \\
{[186-403]}\end{array}$ & $\begin{array}{r}294 \\
{[215-488]}\end{array}$ & 0.18 & 0.40 & 0.51 & $131-320$ \\
\hline Total Bilirubin (mg/dl) & $\begin{array}{r}0.2 \\
{[0.1-0.2]}\end{array}$ & $\begin{array}{r}0.2 \\
{[0.1-0.3]}\end{array}$ & 0.63 & $\begin{array}{r}0.2 \\
{[0.2-0.3]}\end{array}$ & $\begin{array}{r}0.2 \\
{[0.2-0.3]}\end{array}$ & 1.00 & 0.52 & 1.00 & $0.1-0.4$ \\
\hline $\mathrm{ALT}^{\dagger}$ (units/l) & $\begin{array}{r}35 \\
{[17-166]}\end{array}$ & $\begin{array}{r}34 \\
{[23-115]}\end{array}$ & 0.94 & $\begin{array}{r}29 \\
{[17-52]}\end{array}$ & $\begin{array}{r}38 \\
{[16-152]}\end{array}$ & 0.49 & 0.53 & 0.85 & $14-76$ \\
\hline $\mathrm{ALP}^{\ddagger}$ (units/l) & $\begin{array}{r}23 \\
{[10-28]}\end{array}$ & $\begin{array}{r}15 \\
{[9-30]}\end{array}$ & 0.17 & $\begin{array}{r}23 \\
{[12-32]}\end{array}$ & $\begin{array}{r}15 \\
{[6-28]}\end{array}$ & 0.15 & 0.78 & 0.74 & $12-98$ \\
\hline $\mathrm{GGT}^{\S}$ (units/l) & $\begin{array}{r}3 \\
{[3-4]}\end{array}$ & $\begin{array}{r}3 \\
{[3-3]}\end{array}$ & 1.00 & $\begin{array}{r}3 \\
{[3-4]}\end{array}$ & $\begin{array}{r}3 \\
{[3-8]}\end{array}$ & 1.00 & 0.59 & 0.22 & $0-8$ \\
\hline $\mathrm{CK}^{\|}$(units/l) & $\begin{array}{r}64 \\
{[40-109]}\end{array}$ & $\begin{array}{r}97.5 \\
{[48-411]}\end{array}$ & 0.04 & $\begin{array}{r}59.5 \\
{[41-156]}\end{array}$ & $\begin{array}{r}65 \\
{[39-116]}\end{array}$ & 0.79 & 0.96 & 0.05 & 40-226 \\
\hline
\end{tabular}

${ }^{*} P$-values of comparisons between pre-trial and month 4 values.

${ }^{\dagger}$ Alanine aminotransferase.

${ }^{\ddagger}$ Alkaline phosphatase.

$\S \gamma$-glutamyl transferase.

"Creatine kinase.

group decreased $(P<0 \cdot 01)$ slightly by 7,4 and $2 \%$, respectively. The means of erythrocyte counts and haemoglobin concentrations of dogs of the vitamin $\mathrm{D}$ group by the trial end were also less ( -4 and $-2 \%$, respectively) but not significantly. No changes were indicative of adverse health effects and the majority of median values were within the reference interval. Cholesterol and plasma alanine aminotransferase (ALT) values of several dogs were elevated outside the reference interval but were not associated with clinical signs in the dogs.

\section{Plasma $25(\mathrm{OH}) \mathrm{D}_{3}$ status}

Plasma 25(OH) $\mathrm{D}_{3}$ did not differ between groups at the beginning of the study (Table 2). At Part II initiation, canine participants had plasma $25(\mathrm{OH}) \mathrm{D}_{3}$ levels between 13.9 and $36.6 \mathrm{ng} / \mathrm{ml}$. Dogs receiving diet supplemented with $\mathrm{HyD}^{\circledR}$ had an increase in plasma $25(\mathrm{OH}) \mathrm{D}_{3}$ from a mean of 24.4 $\mathrm{ng} / \mathrm{ml}$ to a mean of $34.3 \mathrm{ng} / \mathrm{ml}(P<0 \cdot 05)$ after 1 month (Fig. 1). Plasma $25(\mathrm{OH}) \mathrm{D}_{3}$ concentrations did not significantly change after the initial increase. At the trial end, plasma 25 $(\mathrm{OH}) \mathrm{D}_{3}$ concentrations in dogs that received the $\mathrm{HyD}^{\circledR}$ diet were increased in all but one dog and ranged from a low of $24 \mathrm{ng} / \mathrm{ml}$ to a high of $47 \mathrm{ng} / \mathrm{ml}$. The repeated-measures mixed-model ANOVA when applied with a contrast testing without including initial observations revealed a diet effect $(P=0.044)$. Mean plasma $25(\mathrm{OH}) \mathrm{D}_{3}$ concentrations for dogs ( \pm SEM) supplemented with $\mathrm{HyD}^{\circledR}$ was 33.3 ( $\pm 3.0 \mathrm{ng} /$ $\mathrm{ml}$ ) which was $28 \%$ greater than the mean $25(\mathrm{OH}) \mathrm{D}_{3}$ concentration of dogs supplemented with D3 $(26 \cdot 0 \pm 1.5 \mathrm{ng} / \mathrm{ml})$ 
Table 5. Clinical laboratory urinalysis results of dogs before and 4 months after diet supplementation with vitamin $\mathrm{D} 3(n 8)$ or $\mathrm{HyD}^{\circledR}(n 10)$

\begin{tabular}{|c|c|c|c|c|c|c|c|}
\hline & & \multicolumn{4}{|l|}{ Supplementation } & & \\
\hline & & \multicolumn{2}{|l|}{ Vitamin D3 } & \multicolumn{2}{|l|}{$\mathrm{HyD}^{\circledR}$} & \multicolumn{2}{|c|}{ Vitamin $\mathrm{D} 3 \mathrm{v} . \mathrm{HyD}^{\circledR}$} \\
\hline & & Pre-trial & 4 months & Pre-trial & 4 months & Pre-trial $P$ & 4 months $P$ \\
\hline Specific gravity & MeanRange & $1.032[1.012-1.064]$ & $1.032[1.005-1.053]$ & $1.032[1.004-1.059]$ & $1.038[1.015-1.054]$ & 0.98 & 0.61 \\
\hline \multirow[t]{2}{*}{ Glucose } & Negative $(n)$ & 7 & 8 & 10 & 10 & $\mathrm{nd}^{*}$ & nd \\
\hline & $\geq$ Trace $(n)$ & 0 & 0 & 0 & 0 & & \\
\hline \multirow[t]{2}{*}{ Bilirubin } & Negative/1+ $(n)$ & 7 & 8 & 9 & 9 & 1.00 & 1.00 \\
\hline & $\geq 2+(n)$ & 0 & 0 & 1 & 1 & & \\
\hline \multirow[t]{2}{*}{ Ketones } & Negative/trace $(n)$ & 7 & 8 & 10 & 10 & nd & nd \\
\hline & $>$ Trace $(n)$ & 0 & 0 & 0 & 0 & & \\
\hline \multirow[t]{2}{*}{ Heme } & Negative/trace $(n)$ & 7 & 8 & 10 & 9 & nd & 1.00 \\
\hline & $\geq 1+(n)$ & 0 & 0 & 0 & 1 & & \\
\hline $\mathrm{pH}$ & MedianRange & $6 \cdot 0[5 \cdot 5-7 \cdot 5]$ & $8 \cdot 5[5 \cdot 5-8 \cdot 5]$ & $6 \cdot 5[5 \cdot 5-8 \cdot 5]$ & $6 \cdot 8[5 \cdot 5-8 \cdot 5]$ & 0.49 & 0.26 \\
\hline \multirow[t]{2}{*}{ Protein by dipstick } & Negative $(n)$ & 6 & 5 & 10 & 9 & 0.41 & 0.27 \\
\hline & $\geq 1+(n)$ & 1 & 3 & 0 & 1 & & \\
\hline Urobilinogen & 0.2 & 7 & 8 & 10 & 10 & nd & nd \\
\hline$\left(E U^{\dagger} / d L\right)$ & $>0 \cdot 2$ & 0 & 0 & 0 & 0 & & \\
\hline \multirow[t]{2}{*}{ Leucocyte $\AA^{\ddagger} / \mathrm{hp}^{\S}$} & None/1-3 (n) & 6 & 7 & 9 & 6 & 1.00 & 0.31 \\
\hline & $>3(n)$ & 1 & 1 & 1 & 4 & & \\
\hline \multirow[t]{2}{*}{ Erythrocyte"/hpf } & None/rare $(n)$ & 7 & 7 & 10 & 9 & nd & 1.00 \\
\hline & $\geq 1(n)$ & 0 & 1 & 0 & 1 & & \\
\hline \multirow[t]{2}{*}{ Bacteria/hpf } & None $(n)$ & 7 & 8 & 10 & 9 & nd & 1.00 \\
\hline & Few $(n)$ & 0 & 0 & 0 & 1 & & \\
\hline Transitional Cells/lpf" & None & None & None & None & None & nd & nd \\
\hline \multirow[t]{2}{*}{ Squamous Cells/lpf } & None to $1-5(n)$ & 7 & 8 & 10 & 9 & nd & 1.00 \\
\hline & $>5(n)$ & 0 & 0 & 0 & 1 & & \\
\hline \multirow[t]{2}{*}{ Casts/lpf } & None $(n)$ & 7 & 8 & 10 & 9 & nd & 1.00 \\
\hline & Rare $(n)$ & 0 & 0 & 0 & 1 & & \\
\hline \multirow[t]{4}{*}{ Crystals/lpf } & None $(n)$ & 6 & 5 & 8 & 7 & 1.00 & 1.00 \\
\hline & Moderate/many $(n)$ & 1 & 3 & 2 & 3 & & \\
\hline & Struvite $(n)$ & 1 & 3 & 2 & 2 & & \\
\hline & Other $(n)$ & 0 & 0 & 0 & $1^{a}$ & & \\
\hline
\end{tabular}

* Not determined because of lacking observation in one category.

${ }^{\dagger}$ Ehrlich units.

${ }^{\ddagger}$ Leucocytes.

$\S$ High-power field.

"Erythrocytes.

"Low-power field.

a One dog with 'many struvites and moderate calcium oxalate dehydrate' which had very concentrated urine.

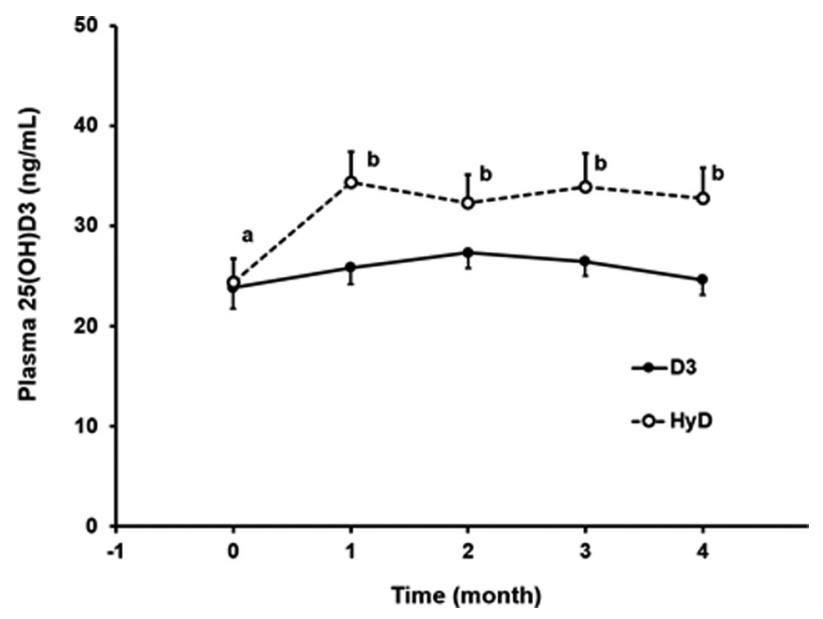

Fig. 1. Plasma $25(\mathrm{OH}) \mathrm{D}_{3}$ concentrations over time for control (D3) and treatment $\left(\mathrm{HyD}^{\circledR}\right)$ groups. All values are means \pm SEMS. Log-transformed data of means of $25(\mathrm{OH}) \mathrm{D}_{3}$ concentrations were compared using repeated-measures ANOVA. Plotted values with different letters indicate significant differences $(P<0.05)$.
(Table 6). For dogs given a diet supplemented with D3, no significant change occurred in plasma $25(\mathrm{OH}) \mathrm{D}_{3}$ concentrations. For these dogs, plasma $25(\mathrm{OH}) \mathrm{D}_{3}$ concentrations at the study end ranged from a low of $19 \mathrm{ng} / \mathrm{ml}$ to a high of $32 \mathrm{ng} / \mathrm{ml}$.

\section{Adverse effects}

There were rarely reported adverse events during the trial. Inappetence following antibiotic treatment of a urinary tract infection occurred in a dog that received the D3-supplemented diet. The dog was subsequently voluntarily withdrawn from the trial. An undesired change in coat colour and consistency was reported by an owner of a dog receiving diet supplemented with $\mathrm{HyD}^{\circledR}$. This change resolved during the trial. Elevation of ALT concentration above the upper limit of the reference interval was revealed via serum biochemistry performed at the trial end in one dog fed the diet supplemented with $\mathrm{HyD}^{\circledR}$. Follow-up biochemistry analysis of this $\operatorname{dog}$ 
Table 6. Statistical significance of effects of supplementation type $\left(\mathrm{HyD}^{\circledR}\right.$ $v$. D3), time of sampling (month), and interaction of effects $\left(\mathrm{HyD}^{\circledR} v\right.$. D3 $\times$ month) on log-transformed plasma 25-hydroxyvitamin D3 concentrations $(\mathrm{ng} / \mathrm{ml})$

\begin{tabular}{lccrrr}
\hline & \multicolumn{2}{c}{ Degrees of freedom } & & \\
\cline { 2 - 3 } Effect & Numerator & Denominator & $F$-value & \multicolumn{1}{c}{$P$} \\
\hline HyD $^{\circledR} v$ v. D3 & 1 & 64 & 2.75 & 0.1022 \\
Month (initial, 1, 2, 3 4) & 4 & 64 & 13.76 & $<0.0001$ \\
HyD $^{\circledR} v$ v. D3 $\times$ Month & 4 & 64 & 4.24 & 0.0042 \\
HyD $^{\circledR}$ v. D3 & NA & 64 & NA & 0.0047 \\
\hline
\end{tabular}

${ }^{\mathrm{a}}$ Baseline removed from values.

showed a reduction of ALT to within the reference interval after 4 months post-trial. One owner reported improvement in stool quality while the dog was fed the D3-supplemented diet. No other notable responses were reported.

\section{Discussion}

We hypothesised that dogs fed a diet supplemented with 25 $(\mathrm{OH}) \mathrm{D}_{3}$ would experience a rapid improvement in vitamin $\mathrm{D}$ status. This effect was anticipated based on previous research in other species, coupled with findings of an acute rise in plasma $25(\mathrm{OH}) \mathrm{D}_{3}$ when purpose-bred dogs were fed treats supplemented with $25(\mathrm{OH}) \mathrm{D}_{3}^{(17,25)}$. In our study, an initial mixed-model ANOVA did not reveal any significant difference in plasma $25(\mathrm{OH}) \mathrm{D}_{3}$ between the dog groups. This result likely reflected the inclusion of $25(\mathrm{OH}) \mathrm{D}_{3}$ observations at time point ' 0 ' in the analysis, during which the dogs had not yet received their assigned experimental diets. This was anticipated as the treatment groups were balanced based on preliminary plasma $25(\mathrm{OH}) \mathrm{D}_{3}$ concentration. A significant group effect was observed once the initial time point was omitted from the statistical analysis (Table 6).

An immediate effect of ingested $25(\mathrm{OH}) \mathrm{D}_{3}$ on plasma $25(\mathrm{OH}) \mathrm{D}_{3}$ was anticipated for several reasons. Absorption of $\mathrm{D} 3$ relative to $25(\mathrm{OH}) \mathrm{D}_{3}$ occurs more slowly, by passive diffusion, largely from intestinal micelles, and possibly through intestinal epithelial cholesterol transporter activity ${ }^{(26)}$. Sufficient amounts of luminal fat, intestinal lipase activity and bile acid concentration are required to form needed micelles. Absorbed D3, which principally enters intestinal lymph free or esterified and incorporated in lipoproteins or bound to plasma proteins, is then sequestered by the liver, adipose, and other tissues ${ }^{(27-29)}$. From the liver, D3 is gradually released after 25 -hydroxylation to $25(\mathrm{OH}) \mathrm{D}_{3}$. Comparatively, alimentary uptake of $25(\mathrm{OH}) \mathrm{D}_{3}$ is more rapid than that of D3 because it has greater water solubility and intestinal protein-binding capabilities ${ }^{(30,31)}$. Alimentary $25(\mathrm{OH}) \mathrm{D}_{3}$ enters circulation more directly relative to $\mathrm{D} 3$, first in portal blood then systemic circulation bound to plasma proteins ${ }^{(32)}$.

Dogs do not respond to supplementation of D3 in high amounts to improve vitamin D status ${ }^{(16,17)}$. Furthermore, dogs and man with liver or gastrointestinal disease have been found insufficient in vitamin $\mathrm{D}$ and may have impairments in vitamin D metabolism that could lower their D status $^{(9,33,34)}$. For these patients, supplementation with $25(\mathrm{OH})$
$\mathrm{D}_{3}$ may be ideal, due to its increased bioavailability to counteract reduced vitamin $\mathrm{D}$ metabolism capabilities.

Another substantive finding was no significant change in plasma $25(\mathrm{OH}) \mathrm{D}_{3}$ concentration for the dogs consuming diet supplemented with D3. The D3 content of the experimental diet (approximately $1000 \mathrm{IU} / \mathrm{Mcal}$, Table 1) was in excess of the current recommended maximum by AAFCO $(750 \mathrm{IU} / \mathrm{Mcal})^{(35)}$. It is less than the prior maximum $(1250$ $\mathrm{IU} / \mathrm{Mcal})$, which several years ago was reduced to the current value to be in accord with NRC safe-upper-limit and FEDIAF Guidelines. The D3 concentration in the experimental diet was likely greater than that of diets dogs were consuming prior to the study, when time 0 plasma $25(\mathrm{OH}) \mathrm{D}_{3}$ concentrations were determined. Kritkos et al. conducted a survey of eighty-one commercially available dog foods and reported a median vitamin D content of $481 \mathrm{IU} / \mathrm{Mcal}$, with no foods in excess of the current $750 \mathrm{IU} / \mathrm{Mcal}^{(8)}$. The D3 content of the experimental diet was comparatively high for dog foods, suggesting that the dogs maintained on the experimental diet were resistant to change in vitamin D status. Heaney et al. suggest that as liver 25-hydroxylases of man become saturated with the D3 substrate, the production of $25(\mathrm{OH}) \mathrm{D}_{3}$ by the liver becomes progressively less influenced by increasingly available D $3^{(29)}$. Hence, resistance to change in vitamin D status with oral vitamin D supplementation should be expected if liver 25-hydroxylase activity is near maximal.

While circulating $25(\mathrm{OH}) \mathrm{D}_{3}$ concentration is a widely accepted indicator of vitamin $\mathrm{D}$ status for many species, specific concentrations of $25(\mathrm{OH}) \mathrm{D}_{3}$ that indicate vitamin $\mathrm{D}$ deficiency, insufficiency, sufficiency and excess are only tentatively agreed upon. An international consensus report on vitamin D status of man recently suggested that serum concentrations of $25(\mathrm{OH}) \mathrm{D}_{3}$ less than $12 \mathrm{ng} / \mathrm{ml}(30 \mathrm{nmol} / \mathrm{l})$ indicate vitamin D deficiency, while concentrations between $20 \mathrm{ng} / \mathrm{ml}(50 \mathrm{nmol} /$ l) and $50 \mathrm{ng} / \mathrm{ml}(125 \mathrm{nmol} / \mathrm{l})$ indicate vitamin $\mathrm{D}$ sufficiency for skeletal health ${ }^{(6)}$. Vitamin D status sufficient for skeletal health maybe insufficient for other health outcomes ${ }^{(36)}$. For example, serum $25(\mathrm{OH}) \mathrm{D}_{3}$ concentrations between 30 and $40 \mathrm{ng} / \mathrm{ml}$ (75-100 nmol/l) may reduce colorectal cancer risk in $\operatorname{man}^{(37)}$. Threshold $25(\mathrm{OH}) \mathrm{D}_{3}$ concentrations and limits for extra-skeletal disease prevention and clinical interventions largely have not been established, although they are being investigated $^{(6)}$. Use of varying methods for analysis of 25 $(\mathrm{OH}) \mathrm{D}_{3}$ have impeded progress; a 'gold standard' methodology is not established. In general, chromatographic methods are less affected by the sample matrix and permit independent quantification D3 and D2 metabolites, and are therefore considered superior to immunoassays ${ }^{(38)}$.

Selting et al. used a competitive chemiluminescence immunoassay in their work and reported serum $25(\mathrm{OH}) \mathrm{D}_{3}$ concentrations of vitamin D sufficiency in dogs to be 100 $120 \mathrm{ng} / \mathrm{ml}$. Our $25(\mathrm{OH}) \mathrm{D}_{3}$ concentrations were lower than theirs. The concentration difference likely reflected assay methodological differences, more than vitamin D deficiency in our dogs. A comparison of results obtained for thirty-three dog serum samples by our HPLC method with the method used by Selting et al. indicates the competitive chemiluminescence immunoassay $25(\mathrm{OH}) \mathrm{D}_{3}$ values are on average greater 
by about $100 \%$ (R. C. B., unpublished results). In using chromatographic methods similar to those used in our study, Horst and Littldike found $25(\mathrm{OH}) \mathrm{D}_{3}$ circulates in many domesticated species between 30 and $50 \mathrm{ng} / \mathrm{ml}^{(39)}$. Our analyses show $25(\mathrm{OH}) \mathrm{D}_{3}$ concentrations ranged from 14 to $59 \mathrm{ng} /$ $\mathrm{ml}$ and had a median value of $34 \mathrm{ng} / \mathrm{ml}^{(39)}$. The aforementioned consensus regarding circulating $25(\mathrm{OH}) \mathrm{D}$ concentrations in man, if speculatively applied to our observations in dogs, indicates that many dogs that we evaluated in the present study, i.e. dogs with $25(\mathrm{OH}) \mathrm{D}_{3}$ concentrations less than 20 $\mathrm{ng} / \mathrm{ml}$, would be considered vitamin D 'insufficient'.

Low initial plasma $25(\mathrm{OH}) \mathrm{D}_{3}$ concentrations in some dogs might have reflected low dietary vitamin $\mathrm{D}$ intake, although the vitamin $\mathrm{D}$ contents of diets historically consumed by the dogs were not determined. At the study end, two dogs that received a diet supplemented with D3 had plasma $25(\mathrm{OH})$ $\mathrm{D}_{3}$ concentrations just below $20 \mathrm{ng} / \mathrm{ml}$. For these dogs, 25-hydroxylation of $\mathrm{D} 3$ to form $25(\mathrm{OH}) \mathrm{D}_{3}$ might have been limited. None of the dogs fed the diet supplemented with $25(\mathrm{OH}) \mathrm{D}_{3}$ had plasma $25(\mathrm{OH}) \mathrm{D}_{3}$ concentrations below 20 $\mathrm{ng} / \mathrm{ml}$ by the study end. Low vitamin $\mathrm{D}$ status of individual dogs, lines of dogs within a breed or dogs of a breed in general as has been reported for immature Great Danes could be rooted in the variation of 25-hydroxylase activity. Liver and kidney microsomal and mitochondrial enzymes with 25-hydroxylase activity (CYP2R1, CYP3A4 and CYP27A) are believed to synthesise $25(\mathrm{OH}) \mathrm{D}_{3}$ from $\mathrm{D} 3^{(40,41)}$. Some polymorphisms of genes encoding the hydroxylases have functional consequences involving vitamin $\mathrm{D}$ metabolism in man ${ }^{(42)}$. Our findings on dietary $25(\mathrm{OH}) \mathrm{D}_{3}$ supplementation demonstrate a means to raise the vitamin $\mathrm{D}$ status of dogs that might have low vitamin D status despite consuming recommended amounts of D3. Besides circumvention of need for $25(\mathrm{OH}) \mathrm{D}_{3}$ synthesis (e.g. as with some liver disease), ingestion of $25(\mathrm{OH}) \mathrm{D}_{3}$ presents a means to correct vitamin $\mathrm{D}$ insufficiency resulting from malabsorption and/or maldigestion of fat that leads to low vitamin D status, as might occur in dogs with inflammatory bowel disease $\mathrm{e}^{(9,33,34)}$.

The use of $\mathrm{HyD}^{\circledR}$ for supplementation was chosen for its demonstrated safety and effectiveness in livestock feeds. $\mathrm{HyD}^{\circledR}$ has been safely and effectively used in poultry feeds for more than 20 years as a vehicle for $25(\mathrm{OH}) \mathrm{D}_{3}$ supplementation. Dietary $25(\mathrm{OH}) \mathrm{D}_{3}$ in place of $\mathrm{D} 3$ improves early skeletal development and immune function in broiler chickens ${ }^{(43)}$. Using $\mathrm{HyD}^{\circledR}$ in swine feeds has been described more recently. Benefits to skeletal health of sows are inferred from findings and greater birth weights of piglets are observed ${ }^{(44)}$. The amount of $25(\mathrm{OH}) \mathrm{D}_{3}$ used in supplementation was much less than that of $\mathrm{D} 3$. Our results indicate that the potency of $25(\mathrm{OH}) \mathrm{D}_{3}$ in the $\mathrm{HyD}^{\circledR}$ supplement was more than five times that of D3 for the dietary matrix studied. A greater potency of $25(\mathrm{OH}) \mathrm{D}_{3}$ relative to $\mathrm{D} 3$ for raising plasma 25 $(\mathrm{OH}) \mathrm{D}_{3}$ concentration is consistent with prior work in dogs and dosage trials in other species $(17,32,43,45,46)$

The HyD ${ }^{\circledR}$ supplementation was well-tolerated in the present study. Clinical haematology and chemistry results were not significantly different with supplementation type; however, subsequent changes may have occurred with a longer study duration. Although significant changes from pre-trial values occurred for some variables, most changes were modest, within clinical laboratory reference intervals (Table 4), and not considered to affect the health of the dogs. The majority of haematologic values were within the reference range (Table 3), with the exception of mean corpuscular volume, which was not considered to be clinically significant. The modest declines in observed haemoglobin, haematocrit and erythrocytes might have reflected environmental exposures of the dogs. The trial was initiated during late fall and winter and concluded during spring and early summer. In captive wolves, declines from winter to summer are reported in haemoglobin, haematocrit, erythrocytes and mean corpuscular haemoglobin concentration ${ }^{(47)}$. The mechanism is speculative but may involve endocrine factors like circulating thyroxine which is found to be greater in winter than in summer in wolves. Biochemical median values were all within reference ranges. Urinalyses were not significantly different, all of which indicated the safety of the diets used. Owners reported no signs of serious health effects. The cause for plasma alanine ALT elevation in one $\operatorname{dog}$ that received the $\mathrm{HyD}^{\circledR}$ diet is unknown. The elevation was not severe and corrected after the dietary change.

Limitations of the present study are important to note. Participants were supplemented for 4 months, and haematologic and biochemical values were not evaluated for most participants once the dogs were transitioned off of the study diet. It is possible that sequelae of vitamin D supplementation occurred following transition off of the study diet. This is less likely given the half-life of $25(\mathrm{OH}) \mathrm{D}_{3}$ appears to be $1 \cdot 8$ weeks in $\operatorname{dogs}{ }^{(17)}$. Even though one breed of dog was used, significant inter-animal variation was sufficient to reduce the group effect, justifying the limitation to one breed. The dogs used in the study were healthy dogs. As previously stated, it is unclear whether vitamin D insufficiency is associated with disease development or contributes to it. Therefore, results may differ when using dogs with various disease processes. While the subjects in this trial were healthy, confirming that a consistent and safe rise in vitamin $\mathrm{D}$ status with $\mathrm{HyD}^{\mathbb{R}}$ supplementation in healthy dogs is an essential first step prior to supplementing patients with concurrent disease processes. Follow-up studies are essential to determine the efficacy of supplementation with $\mathrm{HyD}^{\circledR}$ in improving vitamin $\mathrm{D}$ status in individuals with disease processes associated with vitamin $\mathrm{D}$ insufficiency.

\section{Acknowledgements}

The authors would like to acknowledge Lada Micheas for her statistical assistance. The authors would also like to acknowledge the assistance of the Golden Retriever National Club, Drs Mike Lappin, Shirley Chu, Sessaly Reich and Jeff Bryan for assistance in phlebotomy, and the canine participants, owners and their veterinarians.

This work was supported by the Nestlé Purina Endowed Program in Small Animal Nutrition, in addition to a grant provided by DSM Nutritional Products, LLC. 
Funding was provided by DSM Nutritional Products, LLC. The company also provided the diets for the study.

R. A. K. was the co-investigator of the study, contributed to study design, processed and analysed the data and co-wrote the manuscript. R. C. B. was the principal investigator of the study, contributed to study design, processed and analysed the data and co-wrote the manuscript. S. Y. contributed to study design and reviewed the manuscript.

\section{References}

1. How KL, Hazewinkel HA \& Mol JA (1994) Dietary vitamin D dependence of cat and dog due to inadequate cutaneous synthesis of vitamin D. Gen Comp Endocrinol 96, 12-18.

2. Sharp CR, Selting KA \& Ringold R (2015) The effect of diet on plasma 25-hydroxyvitamin $\mathrm{D}$ concentrations in dogs. BMC Res Notes 8, 442.

3. Stocklin E \& Eggersdorfer M (2013) Vitamin D, an essential nutrient with versatile functions in nearly all organs. Int J Vitam Nutr Res 83, 92-100.

4. Cartwright JA, Gow AG, Milne E, et al. (2018) Vitamin D receptor expression in dogs. J Vet Intern Med 32, 764-774.

5. National Research Council (2006) Nutrient Requirements of Dogs and Cats. Washington, DC: The National Academies Press. https:// doi.org/10.17226/10668.

6. Giustina A, Adler RA, Binkley N, et al. (2020) Consensus statement from 2nd International Conference on Controversies in Vitamin D. Rev Endocr Metab Disord 21, 89-116.

7. Selting KA, Sharp CR, Ringold R, et al. (2016) Plasma 25-hydroxyvitamin D concentrations in dogs - correlation with health and cancer risk. Vet Comp Oncol 14, 295-305.

8. Kritikos G, Weidner N, Atkinson JL, et al. (2018) Quantification of vitamin D3 in commercial dog foods and comparison with Association of American Feed Control Officials recommendations and manufacturer-reported concentrations. J Am Vet Med Assoc 252, 1521-1526.

9. Gow AG, Else R, Evans H, et al. (2011) Hypovitaminosis D in dogs with inflammatory bowel disease and hypoalbuminaemia. $J$ Small Anim Pract 52, 411-418.

10. Kraus MS, Rassnick KM, Wakshlag JJ, et al. (2014) Relation of vitamin D status to congestive heart failure and cardiovascular events in dogs. J Vet Intern Med 28, 109-115.

11. Gerber B, Hassig M \& Reusch CE (2003) Plasma concentrations of 1,25-dihydroxycholecalciferol and 25-hydroxycholecalciferol in clinically normal dogs and dogs with acute and chronic renal failure. Am J Vet Res 64, 1161-1166.

12. Wakshlag JJ, Rassnick KM, Malone EK, et al. (2011) Cross-sectional study to investigate the association between vitamin $\mathrm{D}$ status and cutaneous mast cell tumours in Labrador retrievers. $\mathrm{Br}$ J Nutr 106, S60-S63.

13. Mick PJ, Peng SA \& Loftus JP (2019) Plasma vitamin D metabolites and CXCL10 concentrations associate with survival in dogs with immune mediated disease. Front Vet Sci 6, 247.

14. Jaffey JA, Backus RC, McDaniel KM, et al. (2018) Plasma vitamin D concentrations in hospitalized critically ill dogs. PLoS ONE 13, e0194062.

15. Titmarsh HF, Gow AG, Kilpatrick S, et al. (2015) Low vitamin D status is associated with systemic and gastrointestinal inflammation in dogs with a chronic enteropathy. PLOS ONE 10, e0137377.

16. Young LR \& Backus RC (2016) Oral vitamin D supplementation at five times the recommended allowance marginally affects plasma 25-hydroxyvitamin D concentrations in dogs. J Nutr Sci 5, e31.

17. Young LR \& Backus RC (2017) Plasma 25-hydroxyvitamin $\mathrm{D}_{3}$ and 24R,25-dihydroxyvitamin $\mathrm{D}_{3}$ concentrations in adult dogs are more substantially increased by oral supplementation of 25-hydroxyvitamin $\mathrm{D}_{3}$ than by vitamin $\mathrm{D}_{3}$.J Nutr Sci 6, e30.
18. Bischoff-Ferrari HA, Dawson-Hughes B, Stocklin E, et al. (2012) Oral supplementation with $25(\mathrm{OH}) \mathrm{D}_{3}$ versus vitamin D3: effects on $25(\mathrm{OH}) \mathrm{D}_{3}$ levels, lower extremity function, blood pressure, and markers of innate immunity. J Bone Miner Res 7, 160-169.

19. Cashman KD, Seamans KM, Lucey AJ, et al. (2012) Relative effectiveness of oral 25-hydroxyvitamin D3 and vitamin D3 in raising wintertime serum 25-hydroxyvitamin $\mathrm{D}$ in older adults. Am J Clin Nutr 95, 1350-1356.

20. Heaney RP, Davies KM, Chen TC, et al. (2003) Human plasma 25-hydroxycholecalciferol response to extended oral dosing with cholecalciferol. Am J Clin Nutr 77, 204-210.

21. Schiffman JD \& Breen M (2015) Comparative oncology: what dogs and other species can teach us about humans with cancer. Phil Trans R Soc B 370, 20140231.

22. Torres de la Riva G, Hart BL, Farver TB, et al. (2013) Neutering dogs: effects on joint disorders and cancers in golden retrievers. PLOS ONE 8, e55937.

23. Lensmeyer GL, Wiebe DA, Binkley N, et al. (2006) HPLC method for 25-hydroxyvitamin D measurement: comparison with contemporary assays. Clin Chem 52, 1120-1126.

24. Cazzolli DM, Prittie JE, Fox PR, et al. (2019) Evaluation of serum 25-hydroxyvitamin D concentrations in a heterogeneous canine ICU population. J Vet Emerg Crit Care 29, 605-610.

25. Dusso A, Lopez-Hilker S \& Rapp N (1998) Extra-renal production of calcitriol in chronic renal failure. Kidney Int 34, 368-375.

26. Silva MC \& Furlanetto TW (2018) Intestinal absorption of vitamin D: a systematic review. Nutr Rev 76, 60-76.

27. Fraser DR \& Kodicek E (1968) Enzyme studies on the esterification of vitamin $\mathrm{D}$ in rat tissues. Biochem J 109, 457-467.

28. Tsuprykov O, Chen X, Hocher CF, et al. (2018) Why should we measure free 25(OH) vitamin D? J Steroid Biochem Mol Bio 180, 87-104.

29. Heaney RP, Armas LAG, Shary JR, et al. (2008) 25-Hydroxylation of vitamin D3: relation to circulating vitamin D3 under various input conditions. Am J Clin Nutr 87, 1738-1742.

30. Stamp TCB (1974) Intestinal absorption of 25-hydroxycholecalciferol. Lancet 2, 121-123.

31. Teegarden D, Nickel KP \& Shi L (2000) Characterization of 25-hydroxvitamin D binding protein from intestinal cells. Biochem Biophys Res Commun 275, 845-849.

32. Cesareo R, Falchetti A, Attanasio R, et al. (2019) Hypovitaminosis $\mathrm{D}$ : is it time to consider the use of calcifediol? Nutrients 11, 1016.

33. Allenspach K, Rizzo J, Jergens AE, et al. (2017) Hypovitaminosis D is associated with negative outcome in dogs with protein losing enteropathy: a retrospective study of 43 cases. BMC Vet Rest 13, 96.

34. Khan MA, Dar HA, Baba MA, et al. (2019) Impact of vitamin D status in chronic liver disease. J Clin Exp Hepatol 9, 574-580.

35. Association of American Feed Control Officials Incorporated. AAFCO Official Publication. C2020. Chapter 4. Model Regulations for Pet Food and Specialty Pet Food Under the Model Bill. P141-224.

36. Holick MF (2017) The vitamin D deficiency pandemic: approaches for diagnosis, treatment and prevention. Rev Endocr Metab Disord 18, 153-165.

37. McCullough ML, Zoltick ES, Weinstein SJ, et al. (2019) Circulating vitamin $\mathrm{D}$ and colorectal cancer risk: an international pooling project of 17 cohorts. J Natl Cancer Inst 111, 158-169.

38. Binkley N \& Carter GD (2017) Toward clarity in clinical vitamin D status assessment: $25(\mathrm{OH}) \mathrm{D}$ assay standardization. Endocrinol Metab Clin North Am 46, 885-899.

39. Horst RL \& Littledike ET (1982) Comparison of plasma concentrations of vitamin $\mathrm{D}$ and its metabolites in young and aged domestic animals. Comp Biochem Physiol B Biochem Mol Biol 73, 485-489.

40. Tryfonidou MA, Holl MS, Vastenburg M, et al. (2003) Hormonal regulation of calcium homeostasis in two breeds of dogs during growth at different rates. J Anim Sci 81, 1568-1580.

41. Jolliffe DA, Walton RT, Griffiths CJ, et al. (2016) Single nucleotide polymorphisms in the vitamin D pathway associating with circulating concentrations of vitamin D metabolites and non-skeletal health outcomes: review of genetic association studies. I Steroid Biochem Mol Biol 164, 18-29. 
42. Duan L, Xue Z, Ji H, et al. (2018) Effects of CYP2R1 gene variants on vitamin $\mathrm{D}$ levels and status: a systematic review and meta-analysis. Gene 678, 361-369.

43. Litta $\mathrm{G}$ (2016) Applications of $25 \mathrm{OHD}_{3}$ in poultry meat production. $C A B$ Rev 11, 1-10.

44. Weber GM, Witschi AK, Wenk C, et al. (2014) Triennial growth symposium-effects of dietary 25-hydroxycholecalciferol and cholecalciferol on blood vitamin D and mineral status, bone turnover, milk composition, and reproductive performance of sows. J Anim Sci 92, 899-909.
45. Vaes AMM, Tieland M, de Regt MF, et al. (2018) Dose-response effects of supplementation with calcifediol on serum 25-hydroxyvitamin D status and its metabolites: a randomized controlled trial in older adults. Clin Nutr 37, 808-814.

46. Graeff-Armas LA, Bendik I, Kunz I, et al. (2020) Supplemental 25-hydroxycholecalciferol is more effective than cholecalciferol in raising serum 25 -hydroxyvitamin $\mathrm{D}$ concentrations in older adults. J Nutr 150, 73-81.

47. Seal US \& Mech LD (1983) Blood indicators of seasonal metabolic patterns in captive adult gray wolves. J Wildl Manage 47, 704-715. 